\title{
Boosted static multipole particles as sources of impulsive gravitational waves
}

\author{
J. Podolský* \\ Department of Theoretical Physics, Faculty of Mathematics and Physics, Charles University, V Holešovičkách 2, \\ 18000 Prague 8, Czech Republic \\ J. B. Griffiths ${ }^{\dagger}$ \\ Department of Mathematical Sciences, Loughborough University, Loughborough, Leicestershire LE11 3TU, United Kingdom
}

(Received 23 June 1998; published 18 November 1998)

\begin{abstract}
It is shown that the known solutions for nonexpanding impulsive gravitational waves generated by null particles of arbitrary multipole structure can be obtained by boosting the Weyl solutions describing static sources with arbitrary multipole moments, at least in a Minkowski background. We also discuss the possibility of boosting static sources in (anti-)de Sitter backgrounds, for which exact solutions are not known, to obtain the known solutions for null multipole particles in these backgrounds. [S0556-2821(98)02624-1]
\end{abstract}

PACS number(s): 04.20.Jb, 04.30.Nk

\section{INTRODUCTION}

The purpose of this paper is to clarify the relationship between two classes of exact solutions of Einstein's equations describing different kinds of multipole particles. For static sources, Weyl's class of axially symmetric vacuum solutions is well known [1]. The asymptotically flat solutions of this class can be interpreted as external fields of axisymmetric sources with arbitrary multipole structures (for a thorough review and interpretation see [2] and references therein). For the case of null particles with a multipole structure, exact solutions and the impulsive gravitational waves generated by them have recently been obtained $[3,4]$. In these solutions, the null particles and impulsive waves are taken to propagate in background spaces of constant curvature: namely Minkowski, de Sitter or anti-de Sitter spaces. The question now arises as to what the relations are between these two types of solutions.

It may first be observed that, if the monopole solution is taken to be Schwarzschild, the relation in this case is clear. As shown by Aichelburg and Sexl [5], the impulsive gravitational ( $p p$ ) wave generated by a single null monopole particle can be obtained by boosting a Schwarzschild black hole to the speed of light while its mass is reduced to zero in an appropriate way. Using a similar method, Hotta and Tanaka [6] have boosted the Schwarzschild-de Sitter solution to obtain the spherical gravitational wave generated by a pair of null monopole particles in a de Sitter background. They have also described a similar solution in an anti-de Sitter background. Further details of the boosts and the geometry of the non-expanding wave surfaces formed in these cases have been given elsewhere [7].

It is thus clear that null monopole solutions can be regarded as the limits of static monopole solutions boosted to the speed of light. It would be natural to regard null multipole particles as the limit of static multipole particles boosted in a similar way. For the case of a Minkowski background,

\footnotetext{
*Email address: Podolsky@mbox.troja.mff.cuni.cz

†Email address: J.B.Griffiths@Lboro.ac.uk
}

we will show that this is indeed the case. However, no explicit exact solutions are known which describe static sources of any multipole structure in a background with a non-zero cosmological constant. Such relations in a (anti-)de Sitter background must thus remain unresolved.

\section{MULTIPOLE PARTICLES IN A MINKOWSKI BACKGROUND}

We first consider static axisymmetric solutions with zero cosmological constant. Following Quevedo [2], we use the line element in Weyl coordinates,

$$
\mathrm{d} s^{2}=e^{2 \psi} \mathrm{d} t^{2}-e^{-2 \psi}\left[e^{2 \gamma}\left(\mathrm{d} \varrho^{2}+\mathrm{d} z^{2}\right)+\varrho^{2} \mathrm{~d} \varphi^{2}\right],
$$

where $\psi$ and $\gamma$ are functions of $\varrho$ and $z$ only. In this form, exact asymptotically flat vacuum solutions describing the external fields of sources with a multipole structure in a Minkowski background are given by

$$
\begin{aligned}
& \psi=\sum_{m=0}^{\infty} \frac{a_{m}}{r^{m+1}} P_{m}(\cos \theta) \\
& \gamma=\sum_{m, n=0}^{\infty} \frac{(m+1)(n+1)}{m+n+2} \frac{a_{m} a_{n}}{r^{m+n+2}}\left(P_{m+1} P_{n+1}-P_{m} P_{n}\right) .
\end{aligned}
$$

In this expansion $r=\sqrt{\varrho^{2}+z^{2}}, \cos \theta=z / r$ and $P_{m}$ are the Legendre polynomials with argument $\cos \theta$. The sequence of arbitrary constants $a_{m}$ determine the $m^{\text {th }}$ multipole moments of the source, at least in the Newtonian limit. However, in relativity, the definitions of multipole moments are more complicated (for a full discussion see [2]). In particular, it may be noted that the above "monopole" case in which all $a_{m}$ 's vanish except $a_{0}$ is the Curzon-Chazy solution. As has been shown elsewhere [8] the source at the origin in this case has directional properties and thus a non-spherical structure. On the other hand, the Schwarzschild solution which is the unique asymptotically flat spherically symmetric exterior field for a monopole has the potential $\psi$ for a rod when written in Weyl coordinates. 
We now consider boosting the solutions (2) to the limit in which the speed of the source approaches that of light. As will be clear below, in this limit it is necessary to scale the constants $a_{m}$ to zero in an appropriate way. In this case $\psi$ $\ll 1$, and the function $\gamma$ is of second order and may be neglected. It may be noted that in this approximation we approach the Newtonian limit, and in this case the differences between the Schwarzschild and Curzon-Chazy solutions mentioned above also vanish. Working at this level of approximation, the expansion (2) describes the multipole moments of a source at $r=0$ in a physically meaningful sense.

Introducing standard Cartesian coordinates $x=\varrho \cos \varphi$, $y=\varrho \sin \varphi$, the line element (1) may be written in the approximate form

$$
\mathrm{d} s^{2}=\mathrm{d} t^{2}-\mathrm{d} x^{2}-\mathrm{d} y^{2}-\mathrm{d} z^{2}+2 \psi\left(\mathrm{d} t^{2}+\mathrm{d} x^{2}+\mathrm{d} y^{2}+\mathrm{d} z^{2}\right) .
$$

First we boost this solution in a direction orthogonal to the axis of symmetry which, without loss of generality, may be chosen as the $x$ direction:

$$
t=\frac{\tilde{t}+v \tilde{x}}{\sqrt{1-v^{2}}}, \quad x=\frac{\tilde{x}+v \tilde{t}}{\sqrt{1-v^{2}}} .
$$

In the limit as $v \rightarrow 1$ this yields

$$
\mathrm{d} s^{2}=\mathrm{d} \widetilde{t}^{2}-\mathrm{d} \widetilde{x}^{2}-\mathrm{d} y^{2}-\mathrm{d} z^{2}+4(\mathrm{~d} \tilde{t}+\mathrm{d} \widetilde{x})^{2} \lim _{v \rightarrow 1} \frac{\psi}{1-v^{2}} .
$$

To evaluate this limit, we use the identity (employed elsewhere $[6,7])$

$$
\lim _{v \rightarrow 1} \frac{1}{\sqrt{1-v^{2}}} g(x)=\delta(\tilde{t}+\tilde{x}) \int_{-\infty}^{\infty} g(x) \mathrm{d} x
$$

which is valid in the distributional sense. It is thus necessary to rescale the parameters $a_{m}$ to zero in the same way for each $m$, such that $4 a_{m} / \sqrt{1-v^{2}}=c_{m}$ where $c_{m}$ are a new set of constants which characterize the multipole moments of the boosted source. The result is an impulsive $p p$-wave metric given by

$$
\mathrm{d} s^{2}=\mathrm{d} \widetilde{t}^{2}-\mathrm{d} \widetilde{x}^{2}-\mathrm{d} y^{2}-\mathrm{d} z^{2}+H(y, z) \delta(\tilde{t}+\widetilde{x})(\mathrm{d} \tilde{t}+\mathrm{d} \widetilde{x})^{2}
$$

where

$$
\begin{aligned}
H & =\sum_{m} H_{m} \\
& =\sum_{m} c_{m} \int_{-\infty}^{\infty} \frac{1}{\left(\rho^{2}+x^{2}\right)^{(m+1) / 2}} P_{m}\left(\frac{z}{\sqrt{\rho^{2}+x^{2}}}\right) \mathrm{d} x
\end{aligned}
$$

where $\rho^{2}=y^{2}+z^{2}$.

We observe that, for the simplest case $c_{0} \neq 0, c_{m}=0$ for $m \geqslant 1$ which corresponds to the boosted Curzon-Chazy solution, the integral diverges. However, the divergence can be removed by first making the transformation

$$
\tilde{t}-\tilde{x} \rightarrow \tilde{t}-\tilde{x}+c_{0} \lim _{v \rightarrow 1} \log \left(\tilde{x}+v \tilde{t}-\sqrt{(\tilde{x}+v \tilde{t})^{2}+1-v^{2}}\right)
$$

which gives

$$
\begin{aligned}
H_{0} & =c_{0} \int_{-\infty}^{\infty}\left[\left(\rho^{2}+x^{2}\right)^{-1 / 2}-\left(1+x^{2}\right)^{-1 / 2}\right] \mathrm{d} x \\
& =-2 c_{0} \log \rho .
\end{aligned}
$$

This may be seen to be identical to the Aichelburg-Sexl solution [5] which was originally obtained by boosting the Schwarzschild solution and represents the impulsive gravitational wave generated by a single null (monopole) particle.

For the higher multipole components each term may be considered separately. For arbitrary $m \geqslant 1$, we require to evaluate

$$
H_{m}=c_{m} \int_{-\infty}^{\infty}\left(\rho^{2}+x^{2}\right)^{-(m+1) / 2} P_{m}\left[z\left(\rho^{2}+x^{2}\right)^{-1 / 2}\right] \mathrm{d} x .
$$

Using the standard expression for the expansion of Legendre polynomials we obtain

$$
\begin{aligned}
H_{m}= & c_{m} \sum_{k=0}^{N} \frac{(-1)^{k}(2 m-2 k) !}{2^{m} k !(m-k) !(m-2 k) !} z^{m-2 k} \\
& \times \int_{-\infty}^{\infty}\left(\rho^{2}+x^{2}\right)^{k-m-1 / 2} \mathrm{~d} x
\end{aligned}
$$

where $N=(m-1) / 2$ if $m$ is odd and $N=m / 2$ if $m$ is even. Then, using

$$
\int_{-\infty}^{\infty}\left(\rho^{2}+x^{2}\right)^{-p-1 / 2} \mathrm{~d} x=\frac{2^{2 p}}{p} \frac{(p !)^{2}}{(2 p) !} \frac{1}{\rho^{2 p}} \text { for } p=1,2,3 \ldots
$$

and putting $\cos \phi=z / \rho$ we get

$$
H_{m}(\rho, \phi)=\frac{c_{m}}{\rho^{m}} \sum_{k=0}^{N} \frac{(-1)^{k}(m-k-1) !}{2^{2 k-m} k !(m-2 k) !}(\cos \phi)^{m-2 k} .
$$

Finally, using the identity

$$
\cos m \phi=\sum_{k=0}^{N} \frac{(-1)^{k} m(m-k-1) !}{2^{2 k-m+1} k !(m-2 k) !}(\cos \phi)^{m-2 k}
$$

we obtain that

$$
H_{m}(\rho, \phi)=\frac{2 c_{m}}{m} \frac{\cos m \phi}{\rho^{m}}
$$

This simple term can now be seen to be the $m^{\text {th }}$ multipole component of the exact vacuum solution describing an impulsive gravitational wave with a single source of arbitrary multipole structure as described in [3].

For the sake of completeness, we now consider boosting the metric (3) with the initial static source (2) in the direction of the axis of symmetry, namely in the original $z$ direction. Using similar steps to those above, we obtain 


$$
\mathrm{d} s^{2}=\mathrm{d} \widetilde{t}^{2}-\mathrm{d} x^{2}-\mathrm{d} y^{2}-\mathrm{d} \widetilde{z}^{2}+G(x, y) \delta(\tilde{t}+\widetilde{z})(\mathrm{d} \tilde{t}+\mathrm{d} \widetilde{z})^{2},
$$

with

$$
\begin{aligned}
G & =\sum_{m} G_{m} \\
& =\sum_{m} c_{m} \int_{-\infty}^{\infty} \frac{1}{\left(\rho^{2}+z^{2}\right)^{(m+1) / 2}} P_{m}\left(\frac{z}{\sqrt{\rho^{2}+z^{2}}}\right) \mathrm{d} z
\end{aligned}
$$

where now $\rho^{2}=x^{2}+y^{2}$. For the simplest case, $c_{0} \neq 0, c_{m}$ $=0$ for $m \geqslant 1$, this is identical to the previous case $\left(G_{0}\right.$ $=H_{0}$ ) which yields the Aichelburg-Sexl solution [5]. It is also obvious that $G_{m}=0$ for any odd value of $m$. However, it can also be shown that $G_{m}=0$ for any $m \geqslant 1$. This can be observed by substituting $\xi=z / \sqrt{\rho^{2}+z^{2}}$ so that

$$
\begin{aligned}
G_{m} & =\frac{c_{m}}{\rho^{m}} \int_{-1}^{1}\left(1-\xi^{2}\right)^{m / 2-1} P_{m}(\xi) \mathrm{d} \xi \\
& =\frac{\pi c_{m}}{\rho^{m}} \frac{[\Gamma(m / 2)]^{2}}{\Gamma(m+1 / 2) \Gamma(m / 2+1) \Gamma(1-m / 2) \Gamma(0)}=0,
\end{aligned}
$$

which vanishes since $\Gamma(n)$ diverges at $n=0,-1,-2$, $-3, \ldots$. This is exactly in accordance with intuition since the higher multipoles can be considered in the Newtonian limit as mass distributions (of zero total mass) along the $z$ axis. With a Lorentz contraction in the $z$ direction the effects of these distributions will vanish, leaving only the monopole term $G_{0}$.

\section{MULTIPOLE PARTICLES IN (ANTI-)de SITTER BACKGROUNDS}

In the case of a monopole boosted in the de Sitter or anti-de Sitter backgrounds, exact solutions have already been described in [6] and [7]. These have been obtained by boosting the Schwarzschild-(anti-)de Sitter solution using Lorentz transformations in the five-dimensional representations of the (anti-)de Sitter space-times. By a different method, exact solutions describing impulsive gravitational waves in the de Sitter and anti-de Sitter backgrounds with sources having an arbitrary multipole structure have been given in [4]. It would be of interest to see if these solutions could also be obtained by similarly boosting static multipole sources. Unfortunately, no explicit static solutions are known in space-times with a non-vanishing cosmological constant. However, we wish to make some comments on the kind of solution that could be boosted to give the known exact solutions for null multipole particles.

Of course, it is not possible to use an inverse process of "slowing down" a null particle to obtain a solution for a static particle. We can therefore only speculate on the form of the solution describing a static multipole source in an (anti-)de Sitter background. Nevertheless, there are some clues at least to the linear approximation of the form of such a solution and we will discuss these below.

It seems natural to consider the initial metric to be a per- turbation of (anti-)de Sitter space which reduces to the known form for the monopole case. We therefore assume a line element in the form

$$
\begin{aligned}
\mathrm{d} s^{2}= & \left(1-\epsilon \frac{r^{2}}{a^{2}}\right) \mathrm{d} t^{2}-\left(1-\epsilon \frac{r^{2}}{a^{2}}\right)^{-1} \mathrm{~d} r^{2} \\
& -r^{2}\left(\mathrm{~d} \vartheta^{2}+\sin ^{2} \vartheta \mathrm{d} \varphi^{2}\right)-\psi\left[\mathrm{d} t^{2}+\left(1-\epsilon \frac{r^{2}}{a^{2}}\right)^{-2} \mathrm{~d} r^{2}\right],
\end{aligned}
$$

where $a^{2}=3 /|\Lambda|$ for a cosmological constant $\Lambda, \epsilon=1$ for a de Sitter background $(\Lambda>0), \epsilon=-1$ for an anti-de Sitter background $(\Lambda<0)$, and $\psi$ is a function which is independent of the coordinate $\varphi$ so that the metric (9) is axially symmetric. Note that, as $\Lambda \rightarrow 0$, the line element (9) reduces to a perturbation of Minkowski space-time similar to Eq. (3). For $\psi=2 M / r$ it represents a monopole perturbation corresponding to the Schwarzschild-(anti-)de Sitter solution which was boosted to the ultrarelativistic limit in [6] and [7] to yield exact solutions for impulsive gravitational waves with null monopole sources.

When the perturbation function $\psi$ vanishes, the metric (9) reduces to the line element of the (anti-)de Sitter space-time in static coordinates. It is convenient to express these de Sitter or anti-de Sitter backgrounds as a four-dimensional hyperboloid

$$
Z_{0}{ }^{2}-Z_{1}{ }^{2}-Z_{2}{ }^{2}-Z_{3}{ }^{2}-\epsilon Z_{4}{ }^{2}=-\epsilon a^{2}
$$

embedded in a five-dimensional Minkowski space-time

$$
\mathrm{d} s_{0}^{2}=\mathrm{d} Z_{0}^{2}-\mathrm{d} Z_{1}^{2}-\mathrm{d} Z_{2}^{2}-\mathrm{d} Z_{3}^{2}-\epsilon \mathrm{d} Z_{4}^{2}
$$

where the parametrization is given by

$$
Z_{1}=r \sin \vartheta \cos \varphi, \quad Z_{3}=r \sin \vartheta \sin \varphi, \quad Z_{2}=r \cos \vartheta
$$

and, for $\epsilon=1$,

$$
Z_{0}=\sqrt{a^{2}-r^{2}} \sinh (t / a), \quad Z_{4}= \pm \sqrt{a^{2}-r^{2}} \cosh (t / a)
$$

or, for $\epsilon=-1$,

$$
Z_{0}=\sqrt{a^{2}+r^{2}} \sin (t / a), \quad Z_{4}=\sqrt{a^{2}+r^{2}} \cos (t / a) .
$$

Writing Eq. (9) as $\mathrm{d} s^{2}=\mathrm{d} s_{0}{ }^{2}+\mathrm{d} s_{1}{ }^{2}$, we may express the perturbation as

$\mathrm{d} s_{1}^{2}=-a^{2} \psi\left[\left(\frac{Z_{4} \mathrm{~d} Z_{0}-Z_{0} \mathrm{~d} Z_{4}}{Z_{4}^{2}-\epsilon Z_{0}^{2}}\right)^{2}+\frac{a^{2}}{r^{2}}\left(\frac{Z_{0} \mathrm{~d} Z_{0}-\epsilon Z_{4} \mathrm{~d} Z_{4}}{Z_{4}^{2}-\epsilon Z_{0}^{2}}\right)^{2}\right]$,

where $r=\sqrt{Z_{0}^{2}+\epsilon\left(a^{2}-Z_{4}^{2}\right)}$.

Performing a boost in the $Z_{1}$ direction,

$$
Z_{0}=\frac{\widetilde{Z}_{0}+v \widetilde{Z}_{1}}{\sqrt{1-v^{2}}}, \quad Z_{1}=\frac{\widetilde{Z}_{1}+v \widetilde{Z}_{0}}{\sqrt{1-v^{2}}}
$$

the background is invariant. However, when boosting the source term $\psi$ as above, we must rescale its multiplicative 
constants as $4 a_{m} / \sqrt{1-v^{2}}=c_{m}$. Then, using the identity (4), it can be shown that $\mathrm{d} s_{1}^{2}$ takes the form

$$
\mathrm{d} s_{1}^{2}=-a^{2} H\left(Z_{2}, Z_{4}\right) \delta\left(\widetilde{Z}_{0}+\widetilde{Z}_{1}\right)\left(\mathrm{d} \widetilde{Z}_{0}+\mathrm{d} \widetilde{Z}_{1}\right)^{2}
$$

where

$$
H\left(Z_{2}, Z_{4}\right)=\int_{-\infty}^{\infty} \frac{\epsilon\left(a^{2}-Z_{4}^{2}\right) Z_{4}^{2}+\left(a^{2}+Z_{4}^{2}\right) x^{2}}{\left(Z_{4}^{2}-\epsilon x^{2}\right)^{2}} \frac{\psi}{r^{2}} \mathrm{~d} x
$$

and we must make the substitutions

$$
\begin{gathered}
\cos \vartheta=Z_{2} / r, \quad r=\sqrt{x^{2}+\epsilon\left(a^{2}-Z_{4}^{2}\right)}, \\
\cos [h]^{2}(t / a)=Z_{4}^{2} /\left(Z_{4}^{2}-\epsilon x^{2}\right)
\end{gathered}
$$

in the expression for $\psi / r^{2}$, where $\cos (t / a)$ is used for the case $\Lambda<0$ and $\cosh (t / a)$ for $\Lambda>0$ in the last expression. At this point, we may observe that the structure of the metric perturbation (10) is exactly that required to give the exact null multipole solutions obtained in [4]. These solutions include impulsive gravitational waves located on the null hypersurfaces given by $Z_{2}^{2}+Z_{3}^{2}+\epsilon Z_{4}^{2}=\epsilon a^{2}$, which at any time is a two-sphere in a de Sitter universe or a 2-dimensional hyperboloid in an anti-de Sitter universe as described in [7]. An appropriate parametrization of this wave surface is given by

$$
\begin{aligned}
& Z_{2}=a \sqrt{\epsilon\left(1-z^{2}\right)} \cos \phi, \\
& Z_{3}=a \sqrt{\epsilon\left(1-z^{2}\right)} \sin \phi, \\
& Z_{4}=a z,
\end{aligned}
$$

where $|z| \leqslant 1$ when $\epsilon=1$ and $|z| \geqslant 1$ when $\epsilon=-1$. In terms of these parameters, it was shown in [4] that a general family of vacuum solutions can be expressed in the form

$$
\begin{aligned}
H(z, \phi) & =\sum_{m} c_{m} H_{m}(z, \phi) \\
& =\sum_{m} c_{m} Q_{1}^{m}(z) \cos \left[m\left(\phi-\phi_{m}\right)\right],
\end{aligned}
$$

where $c_{m}$ and $\phi_{m}$ are real constants representing the arbitrary amplitude and phase of each multipole component and $Q_{1}^{m}(z)$ are the associated Legendre functions of the second kind.

Our purpose now is to try to relate the given multipole term $H_{m}(z, \phi)$ of the known exact solution (12) to a possible perturbation term $\psi_{m}$ in Eq. (9). Such a relation is given by the integral (11), but this of course is a non-unique inverse problem.

In order to evaluate the integral (11) and express it in terms of the parameters $z$ and $\phi$, it is convenient to make the substitution from $x$ to $\theta$ such that $x=a \sqrt{\epsilon\left(1-z^{2}\right)} \cot \theta$, after which it takes the form

$$
H(z, \phi)=a\left[\epsilon\left(1-z^{2}\right)\right]^{3 / 2} \int_{0}^{\pi} \frac{z^{2}+\cos ^{2} \theta}{\left(z^{2}-\cos ^{2} \theta\right)^{2}} \frac{\psi(r, \vartheta, t)}{r^{2}} \mathrm{~d} \theta,
$$

in which the coordinates $r, \vartheta, t$ must be expressed using the relations

$$
\begin{gathered}
r=\frac{a \sqrt{\epsilon\left(1-z^{2}\right)}}{\sin \theta}, \quad \cos \vartheta=\sin \theta \cos \phi, \\
\cos [\mathrm{h}]^{2} \frac{t}{a}=\frac{z^{2} \sin ^{2} \theta}{z^{2}-\cos ^{2} \theta} .
\end{gathered}
$$

First we observe that putting $\psi_{0}=a_{0} / r$ gives exactly the monopole case

$$
a^{2} H_{0}=\frac{1}{4} \int_{0}^{\pi} \frac{z^{2}+\cos ^{2} \theta}{\left(z^{2}-\cos ^{2} \theta\right)^{2}} \sin ^{3} \theta \mathrm{d} \theta=\frac{z}{2} \log \left|\frac{1+z}{1-z}\right|-1
$$

as described in [6] and [7]. Second, we observe that the correct dependence of $H_{m}$ on $\phi$ required by Eq. (12) can be obtained if we assume that $\psi_{m}$ is a polynomial of order $m$ in $\cos \vartheta$

$$
\psi_{m}(r, \vartheta, t)=\sum_{n=0}^{m} \chi_{n}^{(m)}(r, t) \cos ^{n} \vartheta
$$

for some suitable functions $\chi_{n}^{(m)}(r, t)$. However, it can be shown that the most natural assumption (2), namely $\psi_{m}=a_{m} r^{-m-1} P_{m}(\cos \vartheta)$, does not yield the required result for $m \geqslant 1$. Instead, for example, the function

$$
\begin{aligned}
\psi_{1}= & 2 \epsilon a_{1} \frac{a^{2} r^{2}}{\sqrt{a^{2}-\epsilon r^{2}}}\left(\frac{r^{2} \cos [h]^{2}(t / a)+3 a^{2} \sin [h]^{2}(t / a)}{r^{4} \cos [h]^{4}(t / a)-a^{4} \sin [h]^{4}(t / a)}\right) \\
& \times \cos [h](t / a) \cos \vartheta
\end{aligned}
$$

does give the correct null dipole term

$$
\begin{aligned}
H_{1} & =Q_{1}^{1}(z) \cos \phi \\
& =-\epsilon \sqrt{\epsilon\left(1-z^{2}\right)}\left(\frac{1}{2} \log \left|\frac{1+z}{1-z}\right|-\frac{z}{z^{2}-1}\right) \cos \phi
\end{aligned}
$$

as given in [4]. This illustrates the fact that an explicit $t$-dependence may be necessary in the linear approximation of the source term in the static background coordinate system. This is unexpected, but may be necessary to maintain the multipole structure in the everywhere curved (anti-)de Sitter background. However, expression (15) may not be a unique linear perturbation term for the non-null dipole. It also diverges in the limit as $\Lambda \rightarrow 0$.

\section{CONCLUSIONS}

For the situation in which the cosmological constant vanishes, we have demonstrated the following results: 
(1) Boosting the asymptotically flat Weyl solutions is an appropriate (but not unique) way of finding exact solutions for null multipole particles.

(2) The solutions obtained in this way are exactly the impulsive $p p$-wave solutions described elsewhere [3].

(3) Boosting the asymptotically flat Weyl solutions in the direction of the axis of symmetry simply yields the Aichelburg-Sexl solution [5] for a null monopole particle.

For the case of a non-zero cosmological constant, no exact solutions for static multipole sources are known. However, solutions for null multipole particles have been obtained [4]. These describe nonexpanding impulsive gravitational waves with null point sources. For the monopole case, the solutions for null particles were obtained by boosting the Schwarzschild-(anti-)de Sitter solution [6,7]. The following points have here been demonstrated:
(1) A simple generalization of the Weyl solutions which describes the field of a static multipole source in an asymptotically (anti-)de Sitter space-time cannot be obtained.

(2) The structure of the known solutions describing null multipole particles in an (anti-)de Sitter universe can be obtained by boosting an appropriate (approximate) multipole solution. However, precise forms of the wave amplitudes are difficult to find.

(3) An exact solution which represents a non-null multipole source in an asymptotically (anti-)de Sitter space-time may have to be non-static.

\section{ACKNOWLEDGMENTS}

This work was supported by the Royal Society and, in part, by grant GACR-202/96/0206 of the Czech Republic and grant GAUK-230/96 of Charles University.
[1] D. Kramer, H. Stephani, M. A. H. MacCallum and E. Herlt, Exact Solutions of Einstein's Field Equations (Cambridge University Press, Cambridge, England, 1980), Sec. 18.1.

[2] H. Quevedo, Fortschr. Phys. 38, 733 (1990).

[3] J. B. Griffiths and J. Podolský, Phys. Lett. A 236, 8 (1997).

[4] J. Podolský and J. B. Griffiths, Class. Quantum Grav. 15, 453 (1998).
[5] P. C. Aichelburg and R. U. Sexl, Gen. Relativ. Gravit. 2, 303 (1971).

[6] M. Hotta and M. Tanaka, Class. Quantum Grav. 10, 307 (1993).

[7] J. Podolský and J. B. Griffiths, Phys. Rev. D 56, 4756 (1997).

[8] S. M. Scott and P. Szekeres, Gen. Relativ. Gravit. 18, 557 (1986). 